\title{
Vestibular Agnosia is linked to abnormal functional brain networks
}

\section{Zaeem Hadi', Yuscah Pondeca ${ }^{1}$, Elena Calzolari', Mariya Chepisheva ${ }^{1}$, Rebecca M Smith $^{1}$, Mohammad Mahmud ${ }^{1}$, Heiko Rust ${ }^{1}$, David J Sharp², Barry M Seemungal ${ }^{1}$}

${ }^{1}$ Brain and Vestibular Group, Neuro-otology Unit, Department of Brain Sciences, Imperial College London.

${ }^{2}$ Computational, Cognitive and Clinical Neuroimaging Laboratory, Department of Brain Sciences, Imperial College London

*Correspondence:

Barry M Seemungal

b.seemungal@imperial.ac.uk

Brain and Vestibular Group

Neuro-otology Unit

Department of Brain Sciences, Imperial College London.

UK. 


\section{Abstract}

Activation of the peripheral vestibular apparatus simultaneously elicits a reflex vestibular nystagmus and the vestibular perception of self-motion (vestibular-motion perception) or vertigo. In a newly characterised condition called Vestibular Agnosia found in conditions with disrupted brain network connectivity, e.g. traumatic brain injury (TBI) or neurodegeneration (Parkinson's Disease), the link between vestibular reflex and perception is uncoupled, such that, peripheral vestibular activation elicits a vestibular ocular reflex nystagmus but without vertigo. Using structural brain imaging in acute traumatic brain injury, we recently linked vestibular agnosia to postural imbalance via disrupted right temporal white-matter circuits (inferior longitudinal fasciculus), however no white-matter tracts were specifically linked to vestibular agnosia. Given the relative difficulty in localizing the neuroanatomical correlates of vestibular-motion perception, and compatible with current theories of human consciousness (viz. the Global Neuronal Workspace Theory), we postulate that vestibular-motion perception (vertigo) is mediated by the coordinated interplay between fronto-parietal circuits linked to whole-brain broadcasting of the vestibular signal of self-motion. We thus used resting state functional MRI (rsfMRI) to map functional brain networks and hence test our postulate of an anterior-posterior cortical network mediating vestibular agnosia. Whole-brain rsfMRI was acquired from 39 prospectively recruited acute TBI patients (and 37 matched controls) with preserved peripheral and reflex vestibular function, along with self-motion perceptual thresholds during passive yaw rotations in the dark, and posturography. Following quality control of the brain imaging, 25 TBI patients' images were analyzed. We classified 11 TBI patients with vestibular agnosia and 14 without vestibular agnosia based on laboratory testing of self-motion perception. Using independent component analysis, we found altered functional connectivity within posterior (right superior longitudinal fasciculus) and anterior networks (left rostral prefrontal cortex) in vestibular agnosia. Regions of interest analyses showed both interhemispheric and intra-hemispheric (left anterior-posterior) network disruption in vestibular agnosia. Assessing the brain regions linked via right inferior longitudinal fasciculus, a tract linked to vestibular agnosia in unbalanced patients (but now controlled for postural imbalance), seed-based analyses showed altered connectivity between higher order visual cortices involved in motion perception and mid-temporal regions. In conclusion, vestibular agnosia in our patient group is mediated by multiple brain network dysfunction, involving primarily left frontal and bilateral posterior networks. Understanding the brain mechanisms of vestibular agnosia provide both an insight into the physiological mechanisms of vestibular perception as well as 
an opportunity to diagnose and monitor vestibular cognitive deficits in brain disease such as TBI and neurodegeneration linked to imbalance and spatial disorientation.

Keywords: vestibular agnosia; self-motion perception; traumatic brain injury; vestibular cognition; vertigo; resting-state functional connectivity.

\section{Introduction}

We recently characterized a clinical syndrome called vestibular agnosia (VA) in patients with acute traumatic brain injury (TBI), where, despite preserved peripheral and reflex vestibular functioning, patients had an attenuated vestibular-mediated sensation of self-motion ('vestibular-motion perception') (Calzolari et al., 2021). Vestibular agnosia has also been recorded in elderly patients, typically with small vessel disease and imbalance (Chiarovano et al., 2016; Imbaud Genieys, 2007; Seemungal, 2006), and in patients with advanced Parkinson's Disease with falls (Yousif et al., 2016). The clinical consequences of VA, in which there is an uncoupling of symptomatic complaint from objective deficit, include impaired recognition by clinicians of easily treatable balance diagnoses in acute cases (due to patients' lack of vertigo complaint), and reduced reliability of a retrospective clinical history which also impedes accurate diagnosis in the chronic setting (Seemungal, 2016). We also noted that TBI patients with VA showed greater postural instability than TBI cases without VA, and combined with the previous report in PD patients with imbalance, suggests a link between VA and imbalance (Calzolari et al., 2021; Yousif et al., 2016).

We recently showed that in acute TBI patients with imbalance, objective laboratory-obtained measures of VA correlate with impaired brain structural integrity in the white matter (identified using diffusion tensor imaging - DTI) in the right inferior longitudinal fasciculus (ILF) (Calzolari et al., 2021). This DTI analysis thus identified the overlap in imbalance and VA, however the neural correlates distinct to VA were not identified since a whole brain correlation with VA scores did not reach significance. One explanation is that our study was not appropriately powered - despite the very large clinical effect size - to detect the damaged white matter integrity mediating VA. Another explanation is that VA could arise from disconnections in separate (i.e. non-overlapping) hubs in the whole brain connectome. Put another way, VA is not linked to damage in a unique brain region but could arise from damage to a number of distinct brain networks. 
There is evidence to support the notion that self-motion perception is not localizable. We previously showed that the duration of vertigo sensation in the dark (elicited by a rapid stop from constant angular rotation) in healthy individuals correlated with a widespread, bilateral white matter network (Nigmatullina et al., 2015). Subsequent tractography studies also showed that vestibular networks are bihemispheric linked via the corpus callosum (Kirsch et al., 2016; Wirth et al., 2018). In support of a diffused coding for self-motion perception is the lack of effect of acute focal stroke (or focal neurostimulation) on vestibular-motion perception (Kaski et al., 2016; Seemungal et al., 2008).

Perhaps significantly, although we found that most TBI patients with VA were unbalanced, some patients with severe VA had normal balance, indicating that there are at least two distinct hubs linked to VA, i.e., one related to postural control and one which is unrelated to postural control (Calzolari et al., 2021). Assuming that VA in our cohort is not linked to disconnection of ascending vestibular signals to cortical level (on the basis that such an anatomical disconnection at thalamic level would be associated with severe disability incompatible with laboratory assessment), it follows that a parsimonious hypothesis underlying VA suggests that there are (at least) two main network hubs linked to VA; a posterior brain network which receives the main bottom-up vestibular signals that mediates a sensory processing and sensorimotor functions (e.g. postural control, spatial orientation), and an anterior network linked to perceptual ignition (Del Cul et al., 2007). Thus, dysfunction of the posterior network should always be linked to loss of perception via impaired afferent signal processing and associated sensorimotor impairment (e.g. imbalance); whereas dysfunction of the anterior ‘ignition' network mediating vestibular perceptual ignition will mediate VA but not necessarily sensorimotor dysfunction (if the posterior network is intact). Obviously, most patients with severe TBI with widespread disconnection will manifest combined deficits. We thus postulate that vestibular-motion perception (vertigo), is mediated by the coordinated interplay between fronto-parietal circuits linked to whole-brain broadcasting of the vestibular signal of selfmotion.

To test our postulate of an anterior-posterior cortical network mediating vestibular agnosia, we hypothesize that VA in acute TBI is mediated by multi-network dysfunction, i.e. (i) by impaired functional connectivity within anterior networks, (ii) impaired functional connectivity within posterior networks, and (iii) inter-network impaired functional connectivity. We further hypothesize that posterior networks containing putative vestibular regions (parieto-insular vestibular cortex, mid temporal regions) will have disrupted functional projections. Finally, 
since we previously showed that VA was linked to imbalance via damage to the right inferior longitudinal fasciculus (ILF) (Calzolari et al., 2021) and ILF connects higher order visual cortices to the temporal regions (Latini et al., 2017; Panesar et al., 2018), we probed the cortical regions mapped by right ILF using lingual gyrus as seed, which is often linked to motion- and illusion-perception (Cha \& Chakrapani, 2015; Della-Justina et al., 2014; Kahane et al., 2003; Roberts et al., 2017). To assess our predictions, we evaluated functional connectivity via resting state fMRI (rsfMRI) in both grey and white matter brain networks.

\section{Material and Methods}

\subsection{Participants and Recruitment}

The data reported in this paper was collected as part of an MRC-funded prospective study (Calzolari et al., 2021). Thirty-nine acute TBI patients were recruited from the St Mary's Hospital Major Trauma Centre (London, UK) and King's College Hospital (London, UK). Inclusion Criteria were: (i) blunt head injury resulting in admission to the major trauma ward; (ii) age 18-65; (iii) preserved vestibular function. Exclusion criteria were: (i) additional active pre-morbid medical, neurological, or psychiatric condition (unless inactive or controlled); (ii) musculoskeletal condition impairing ability to balance; (iii) substance abuse history; (iv) pregnancy; and (v) inability to obtain consent or assent. Thirty-seven matched healthy controls were also recruited following written informed consent. The study was conducted in accordance with the principles of the Declaration of Helsinki and was approved by the local Research Ethics Committee.

\subsection{Procedure}

All participants completed assessment of peripheral and reflex vestibular function, vestibular perceptual testing, reaction times, posturography, neuroimaging, and questionnaires for perceived balance, and cognitive examination. The details for all tests has been reported previously (Calzolari et al., 2021). Procedures involved in assessment of peripheral vestibular dysfunction, vestibular perceptual testing, posturography, and resting-state scans are listed here.

\subsection{Assessment of Peripheral Vestibular Function}

Patients were assessed for their peripheral vestibular function to exclude peripheral dysfunction as a cause of impaired vestibular perception. Video head impulse testing and rotational chair testing with eye movement assessment of VOR gain for the stopping response from $90 \% \mathrm{~s}$ 
constant rotation was used to assess peripheral dysfunction. All TBI patients included in the study had intact peripheral vestibular function (Calzolari et al., 2021).

\subsection{Vestibular Perceptual Threshold}

Vestibular perceptual thresholds were determined as a measure of self-motion perception and to classify subjects having abnormal perceptual thresholds with VA (Calzolari et al., 2021). Figure 1 shows the apparatus used to determine participants' vestibular-perceptual thresholds during passive yaw-plane rotations in the dark (Seemungal et al., 2004). Participants sat on a rotating chair in dark and were instructed to press right or left button as soon as they perceive the rotation in respective direction (Figure 1A and Figure 1B). Lights were turned on after each trial in order to allow the decay of post-rotatory vestibular effects. White noise was provided via 2 speakers (for each ear) attached with the chair to mask any auditory cues from the environment.

\section{A}

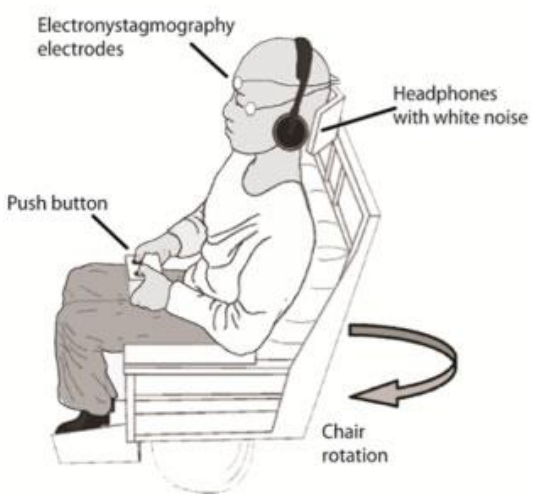

B

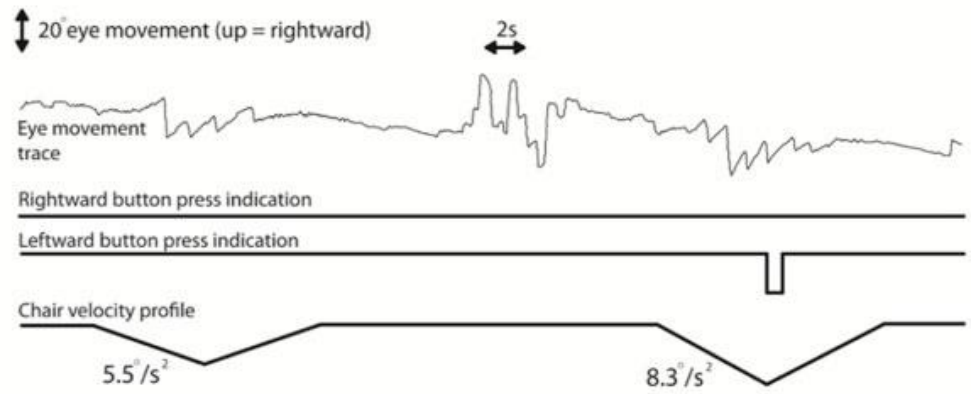

Figure 1. Vestibular thresholds. Apparatus. (A) Rotating Chair. (B) Raw traces for two subsequent rotations for a patient.

\subsection{Posturography}

Postural sway was assessed using a force platform for 60 seconds duration under four conditions: hard surface with eyes open (HO), hard surface with eyes closed (HC), soft surface with eyes open (SO), and soft surface with eyes closed (SC). Subjects were instructed to stand with their arms hanging loosely and to maintain their balance. SC condition is primarily vestibular dependent due to reduced proprioceptive (soft surface) and visual feedback (eyes closed); SC has also been shown to be the condition that best predicts difference in balance between TBI patients and healthy controls (Calzolari et al., 2021). Root mean square (RMS) sway calculated using custom MATLAB scripts, during SC condition was thus chosen as the 
covariate for removing the confounding effect of balance from motion perception in neuroimaging analysis.

\subsection{Vestibular Agnosia Classification}

Vestibular perceptual thresholds from 37 healthy participants were used to establish a normative range. We have previously used a more conservative approach by classifying patients with having VA if their mean perceptual threshold (average of thresholds in right and left direction rotations) were above the mean +3 standard deviation of the perceptual thresholds of healthy controls (Calzolari et al., 2021). This criterion enhances specificity but at the cost of reduced sensitivity. We thus used a slightly lenient and arguably objective approach for classification using all 76 subjects (healthy: 37; TBI: 39) through k-means clustering. We identified 3 clusters (Figure 2): i) cluster 1 was composed of subjects with normal perceptual thresholds (labeled as healthy controls and normal TBI in Figure 2); ii) cluster 2 was composed of subjects with moderately high perceptual thresholds (labeled as healthy outliers and moderate VA TBI in Figure 2), and iii) cluster 3 was composed of subjects with high perceptual thresholds (labeled as high VA TBI in Figure 2). 19 TBI patients from cluster 2 and 3 with moderately high and high perceptual thresholds respectively, were classified as having vestibular agnosia (VA+) whereas 20 TBI patients clustered with the healthy subjects in cluster 1 were considered patients without vestibular agnosia (VA-). Healthy control data was not used in the imaging analysis (and were used only in supporting the classification of vestibular agnosia). Imaging analysis compared TBI patients without vestibular agnosia (VA-) who were considered the control group, with TBI patients with vestibular agnosia (VA+). 


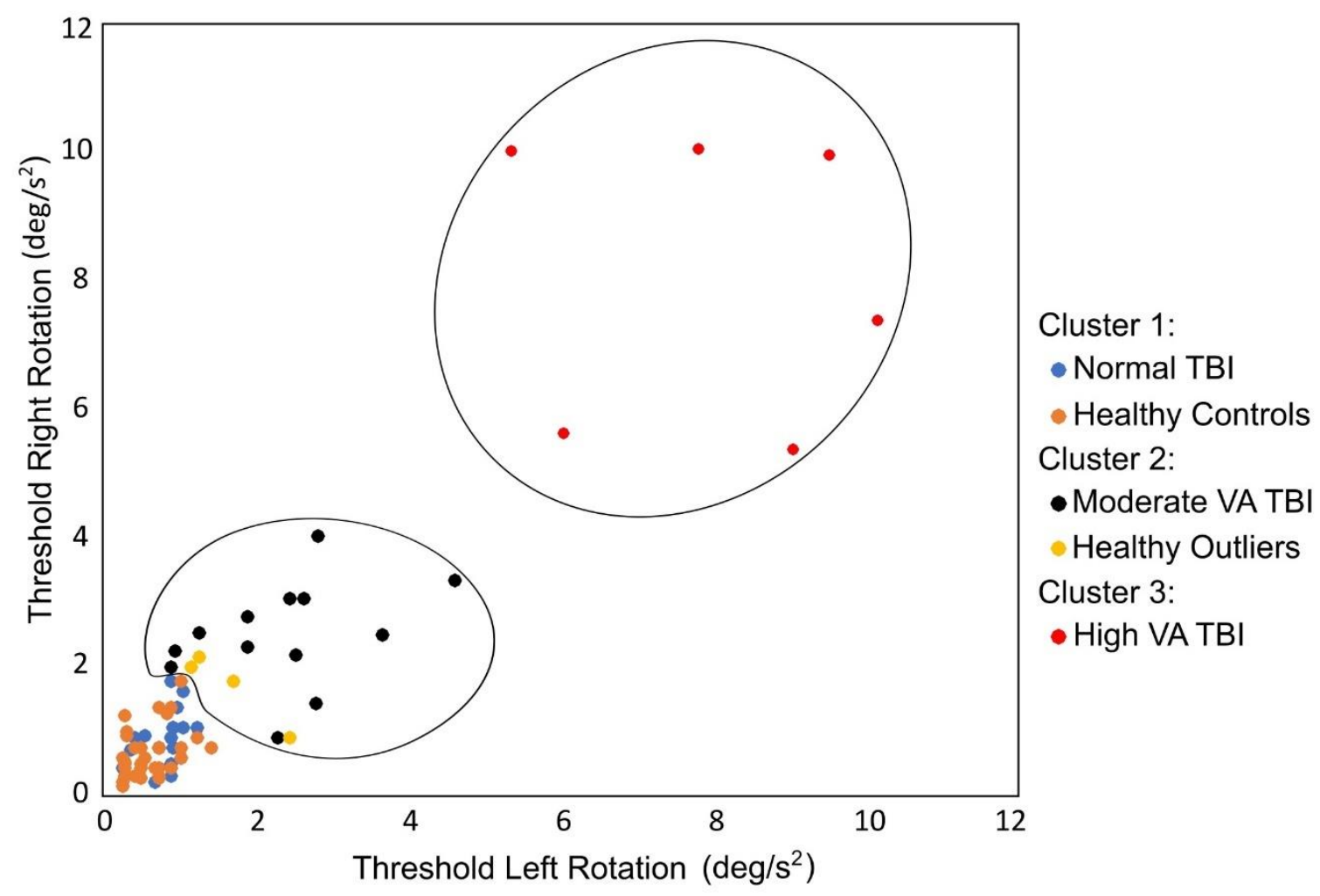

Figure 2. K-means clustering with 3 clusters. K-mean clustering was performed on subjects' perceptual thresholds on right and left sides and three clusters were determined.

\section{Neuroimaging}

Historically, fMRI studies have assessed activity in grey matter (Ogawa et al., 1992) whereas fMRI activity within the white-matter was considered noise. However, recent evidence suggests that the BOLD signal in white matter has similar hemodynamic response characteristics as in grey matter (Fraser et al., 2012) and the functional activity in underlying white-matter tracts maps along the direction of white-matter tracts (Ding et al., 2013, 2016). Several studies have reported the presence of a white-matter functional architecture corresponding to resting state functional activity (Huang et al., 2020; Li et al., 2020; Peer et al., 2017) as well as task related activity (Huang et al., 2018, 2020; Marussich et al., 2017).

We thus performed neuroimaging analysis in grey- and white-matter regions separately using resting-state fMRI scans. After excluding 14 of 39 TBI patients due to different field of view scanning parameters $(n=12$ of 39$)$, excessive motion artifacts $(n=1$ of 39$)$, and failed segmentation ( $\mathrm{n}=1$ of 39 ), we were left with 25 TBI patients (11 VA+ and 14 VA-) whose data were included in the neuroimaging analysis. 


\subsection{Image Acquisition}

Structural and functional MRI images were acquired using a 3T Siemens Verio (Siemens) scanner, using a 32-channel head coil. The scanning protocol included: (i) 3D T1-weighted images acquired using MPRAGE sequence (image matrix: 256 x 256; voxel size: 1 x 1 x 1 $\mathrm{mm}^{3}$; Slices: 160; field of view: 256 x $256 \mathrm{~mm}$; slice thickness: $3 \mathrm{~mm}$; TR = $2300 \mathrm{~ms}$; TE: 2.98 $\mathrm{ms}$ ); and (ii) $\mathrm{T} 2 *$-weighted images sensitive to blood oxygenation level dependent (BOLD) signal for resting state fMRI (image matrix: 64 x 64; voxel size: 3 × 3 × $3 \mathrm{~mm}^{3}$; Slices: 35; field of view: 192 x $192 \mathrm{~mm}$; flip angle: $80^{\circ}$; slice thickness: $3 \mathrm{~mm}$; TR = $2000 \mathrm{~ms}$; TE: $30 \mathrm{~ms}$; volumes $=300$; scan time $=10$ minutes). Subjects were instructed to keep their eyes closed, stay awake, and to try not to think of anything.

\subsection{Preprocessing}

Data were preprocessed using the CONN Toolbox (Whitfield-Gabrieli \& Nieto-Castanon, 2012) based on Statistical Parametric Mapping (SPM12; http://www.fil.ion.ucl.ac.uk/spm/). Preprocessing steps were as follows. (1) Realignment to mean functional image, unwarping, and susceptibility distortion correction. (2) Slice timing correction. (3) Functional outlier detection using ART (artifact detection toolbox), with scans exceeding framewise displacement of $0.5 \mathrm{~mm}$ were labeled as outliers (Power et al., 2012). (4) Structural segmentation and normalization. (5) Functional normalization using deformation fields estimated from structural normalization. (6) Semi-automated lesion masking using T1 images with ITK-SNAP software (Yushkevich et al., 2006) and subtraction of lesions from individual participants' segmented maps. (7) Group analysis space masks: two analysis space masks were created: 1) grey-matter (GM) specific; 2) white-matter (WM) specific. Segmented masks of individual participants were intersected with functional masks to remove regions with missing functional data. These individual masks were binarized (GM: $p>0.2 ; \mathrm{WM}: \mathrm{p}>0.8$ ) and then averaged across subjects. Voxels identified in $>70 \%$ subjects were used for GM specific mask creation whereas voxels in $>90 \%$ were selected for WM specific mask creation. (8) Lesion masks of all subjects were then combined and subtracted from the two group analysis space masks. (9) Smoothing (6 mm FWHM) was performed within the grey- and white-matter using GM and WM masks separately. (10) Subsequently, denoising was performed using aCompCor (Behzadi et al., 2007) approach as implemented in CONN, in which six motion regressors (3 translational and 3 angular motion), their temporal derivatives, cerebrospinal fluid (10 principal components), and outlier scans identified by ART toolbox were regressed out. One subject with $>50 \%$ (199 of 300) outlier scans was removed from the analysis. (11) Data was then band-pass 
filtered with frequency range of $0.008-0.1 \mathrm{~Hz}$. White matter activity and the global signal were not regressed out since white-matter activity was the signal of interest whereas the global signal regression is known to introduce negative correlations (Murphy et al., 2009).

\subsection{Second-Level Analysis}

Group level analysis was performed to determine the differences between VA+ $(n=11)$ TBI patients and VA- $(n=14)$ TBI patients. RMS sway and lesion volume were added as covariates in group-analysis to remove the effects explained by balance and extent of injury. In addition, volume regressors were also added to control for tissue atrophy as a result of injury. Grey matter volume was added as a covariate in GM specific whereas white matter volume was added in WM specific analysis.

\subsubsection{Independent Component Analysis}

Group ICA was performed to assess the intra-network resting state differences in VA+ and VA- groups using Fast ICA algorithm in CONN toolbox (Whitfield-Gabrieli \& NietoCastanon, 2012). The optimal number of the independent components (ICs) were estimated in the GIFT toolbox (https://trendscenter.org/software/) using a modified minimum description length algorithm (MDL). The optimal number of ICs for GM specific analysis were found to be 38 and 10 for WM specific analysis.

In GM specific ICA, independent components containing putative vestibular regions (i.e. parietal operculum (OP2), temporo-parietal, mid temporal, thalamus, and insular regions) were considered components of interest and were evaluated for group comparisons (VA+ vs VA-). All 10 independent components were evaluated for group comparisons in WM specific ICA.

\subsubsection{Region of Interest Analysis}

ROI Analysis was used to assess the inter-network resting state differences between VA+ and VA-groups. To select the network ROIs, we used the dice coefficient, which is a measure of spatial overlap between two images. The dice coefficient was measured for overlap between standard atlases and the independent components from our GM- and WM-specific ICA. For GM specific ROI analysis, we used an atlas of resting state networks available with CONN toolbox, determined using Human Connectome Project dataset of 497 subjects. Resting state network ROIs with dice-coefficient $\geq 0.1$ (25 ROIs) were included in GM specific ROI analysis. Grey-matter resting state networks have been shown to have the dice-coefficients greater than 0.1 (Branco et al., 2016, 2020; Tie et al., 2014). 
Since there are no established white-matter resting-state networks, white matter regions from the ICBM-DTI-81 atlas (Mori et al., 2008; Oishi et al., 2008) were used as ROIs. The regions with the dice-coefficient $\geq 0.01$ (29 ROIs) were included in WM specific ROI analysis. As only 10 independent components were estimated in WM specific ICA, the components were not sufficient to resolve all white-matter regions available in the ICBM-DTI-81 atlas with a considerable value of the dice-coefficient. Thus, we used a lenient cut-off for the dicecoefficient in WM specific ROI analysis to include more white-matter regions in the analysis.

\subsubsection{Seed-Based Analysis}

For testing the hypothesis that posterior networks containing putative vestibular regions will have disrupted functional projections, we selected 3 resting-state networks determined via GM specific ICA as seeds (supplementary Figure S1). All three networks contained regions from PIVC (parietal operculum), mid temporal, and insular regions. Furthermore, we probed the brain regions mapped by ILF using bilateral lingual gyri as seeds using default atlas of CONN, which is a combination of the Harvard-Oxford atlas and the AAL atlas.

\section{Statistical Analysis}

The assumptions of gaussian random field theory (RFT) are required for whole brain analysis (Nichols, 2012) and thus parametric RFT statistics are not applicable for GM or WM specific analysis. Permutation statistics (non-parametric), however, does not require gaussian RFT assumptions (Zhang et al., 2009). Moreover, the choice of cluster height threshold (either $\mathrm{p}<0.01$ or $\mathrm{p}<0.001$ ) has no impact on false positive rate when using permutation statistics for whole brain analysis as demonstrated in a previous study (Eklund et al., 2019).

Thus, all group level differences in ICA and seed-based analysis were evaluated using permutation statistics (Bullmore et al., 1999) and cluster mass FDR correction as implemented in the CONN toolbox. Cluster height threshold for all grey-matter specific analysis was selected as $\mathrm{p}<0.001$ and for all white-matter specific analysis as $\mathrm{p}<0.01$, as choice of height thresholds reveal different architecture in grey- and white-matter.

For WM specific ROI analysis, a connection level threshold of $\mathrm{p}<0.01$ and ROI level FDR mass correction was used. For GM specific ROI analysis, we used a parametric multivariate pattern analytic (MVPA) measure called "Functional Network Connectivity" (Jafri et al., 2008) implemented in the CONN toolbox. 
bioRxiv preprint doi: https://doi.org/10.1101/2021.12.03.471139; this version posted December 7, 2021. The copyright holder for this preprint (which was not certified by peer review) is the author/funder, who has granted bioRxiv a license to display the preprint in perpetuity. It is made available under aCC-BY 4.0 International license.

\section{Results}

\subsection{Independent Component Analysis}

\subsubsection{GM Specific Independent Component Analysis}

Group differences were identified in IC-8, a resting-state network largely composed of bilateral superior and mid temporal regions of posterior division and of temporooccipital part (Figure 3A). VA+ group compared to VA- group had decreased functional connectivity at right intracalcarine cortex (MNI coordinates: 06, -72, 12) in IC-8, shown in Figure 3B $(\mathrm{t}(20)=-4.42$, pFDR < 0.05; effect size $=-3.79$ ).

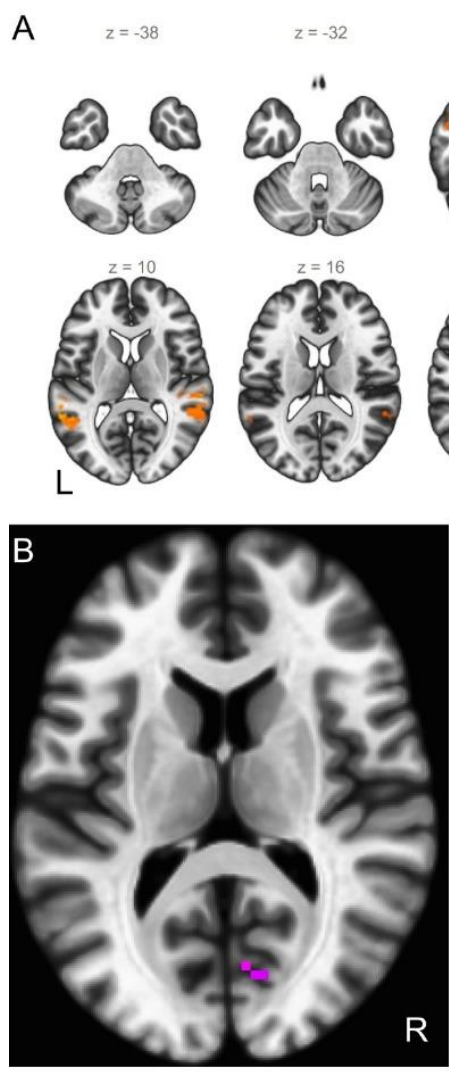

$z=12$
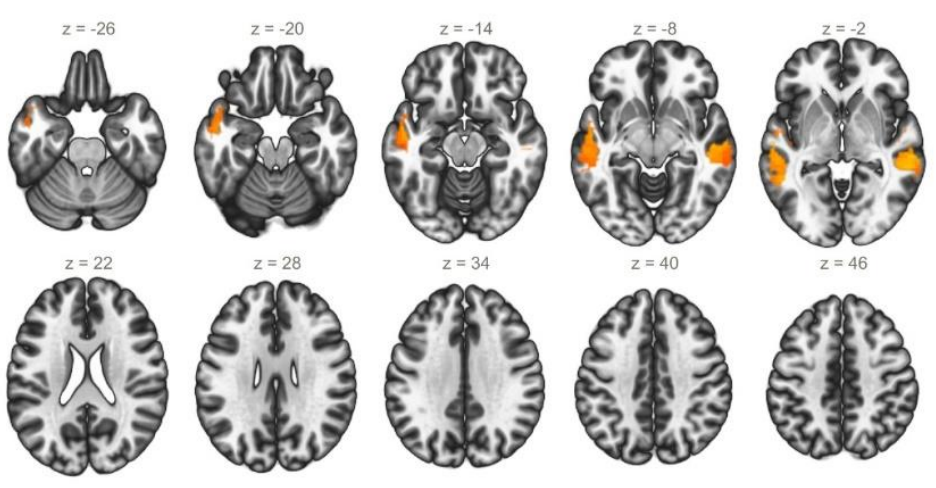

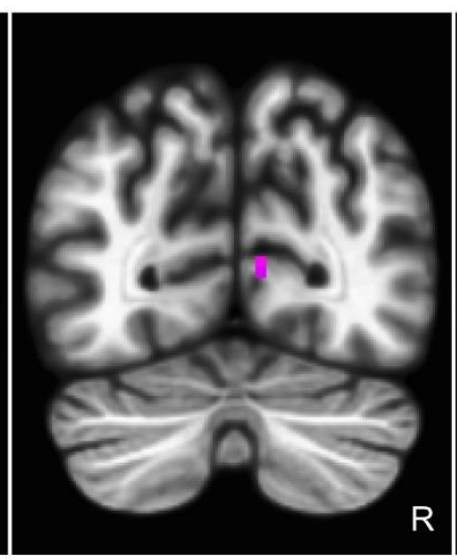

$y=-72$

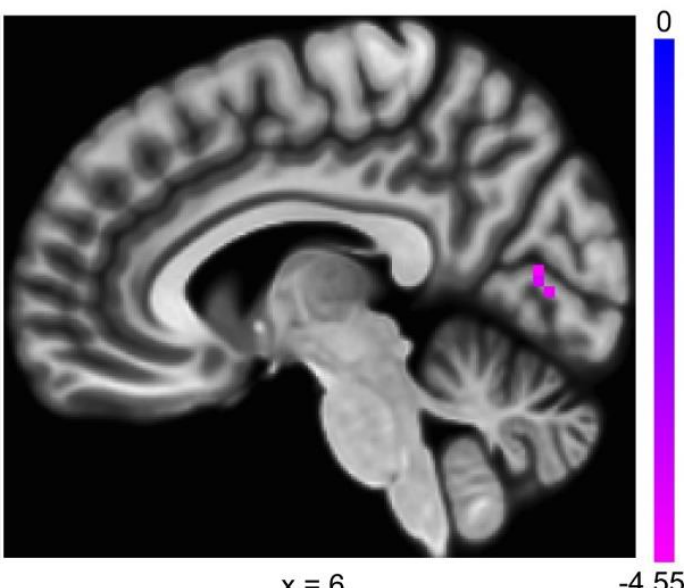

$x=6$

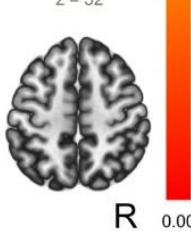

$\mathrm{R} 0.00$
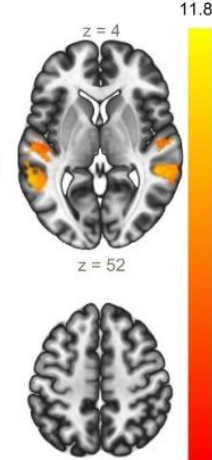

$-4.55$

Figure 3. GM Specific ICA. (A) Independent component 8. (B) Group comparison between $\mathrm{VA}+$ and VA- patients showing decreased functional connectivity in right intra-calcarine cortex inside the independent component-8.

Additionally, group differences were also identified in IC-18, a resting state-network composed of regions from bilateral rostral prefrontal cortex, bilateral hypothalamus and thalamus (Figure 4A). VA+ group compared to VA- group had increased functional connectivity at left frontal pole (MNI coordinates: $-42,48,00)$ in IC-18, shown in Figure 4B $(\mathrm{t}(20)=5.43$, pFDR $<0.05$; effect size $=4.582$ ). 


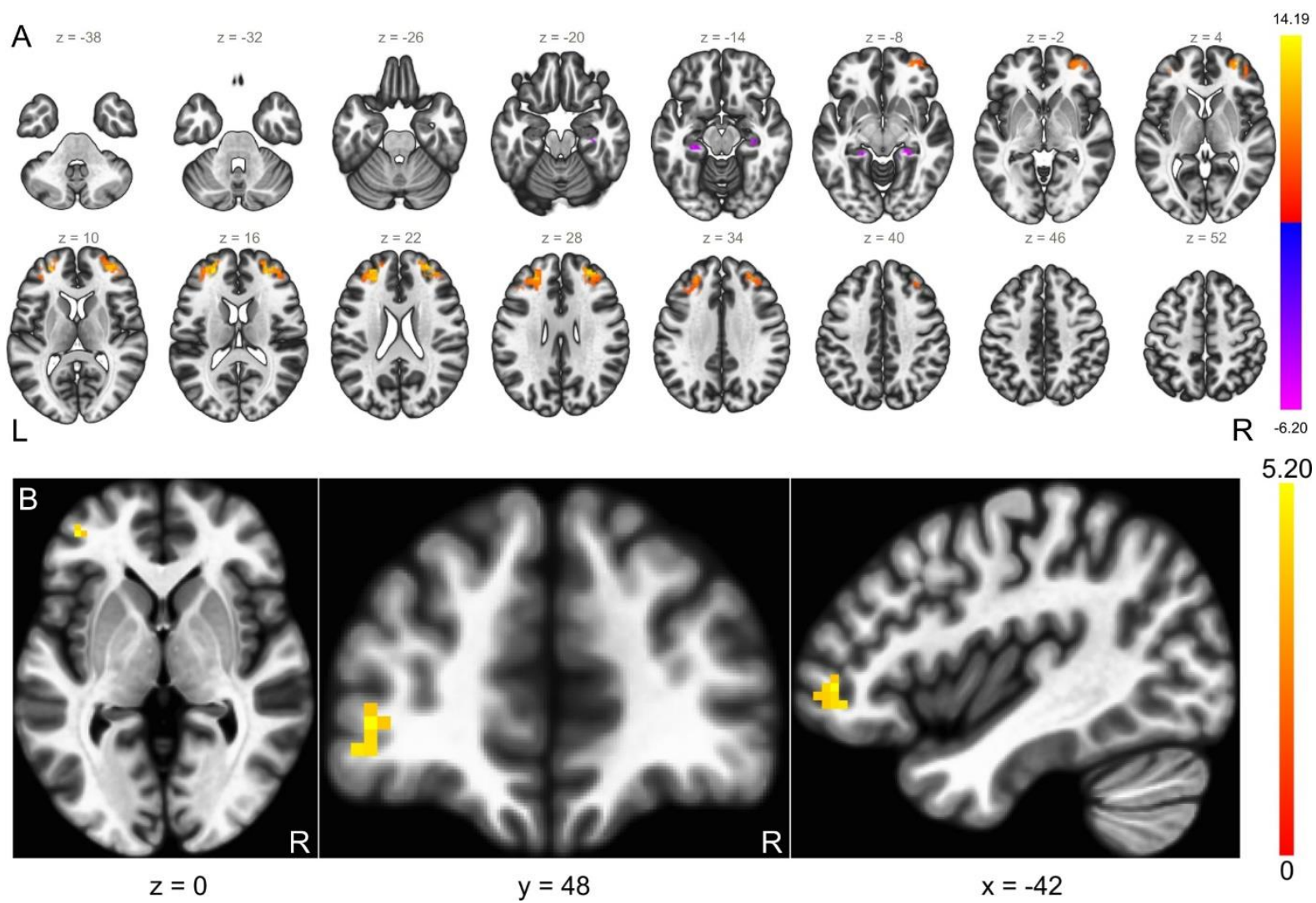

Figure 4. GM Specific ICA. (A) Independent component 18. (B) Group comparison between $\mathrm{VA}+$ and VA- patients showing increased functional connectivity at left frontal pole inside the independent component- 18 .

\subsubsection{WM Specific Independent Component Analysis}

In IC-8 (Figure 5A), we found that VA+ compared to VA- group had increased functional connectivity $(\mathrm{t}(20)=6.34, \mathrm{pFDR}<0.05$; effect size $=1.629)$ in a cluster $(\mathrm{MNI}: 39,-51,06)$ composed of right superior longitudinal fasciculus (SLF), right posterior thalamic radiation (PTR), right retrolenticular part of internal capsule (RLIC), and right sagittal stratum (SS), shown in (Figure 5B). 


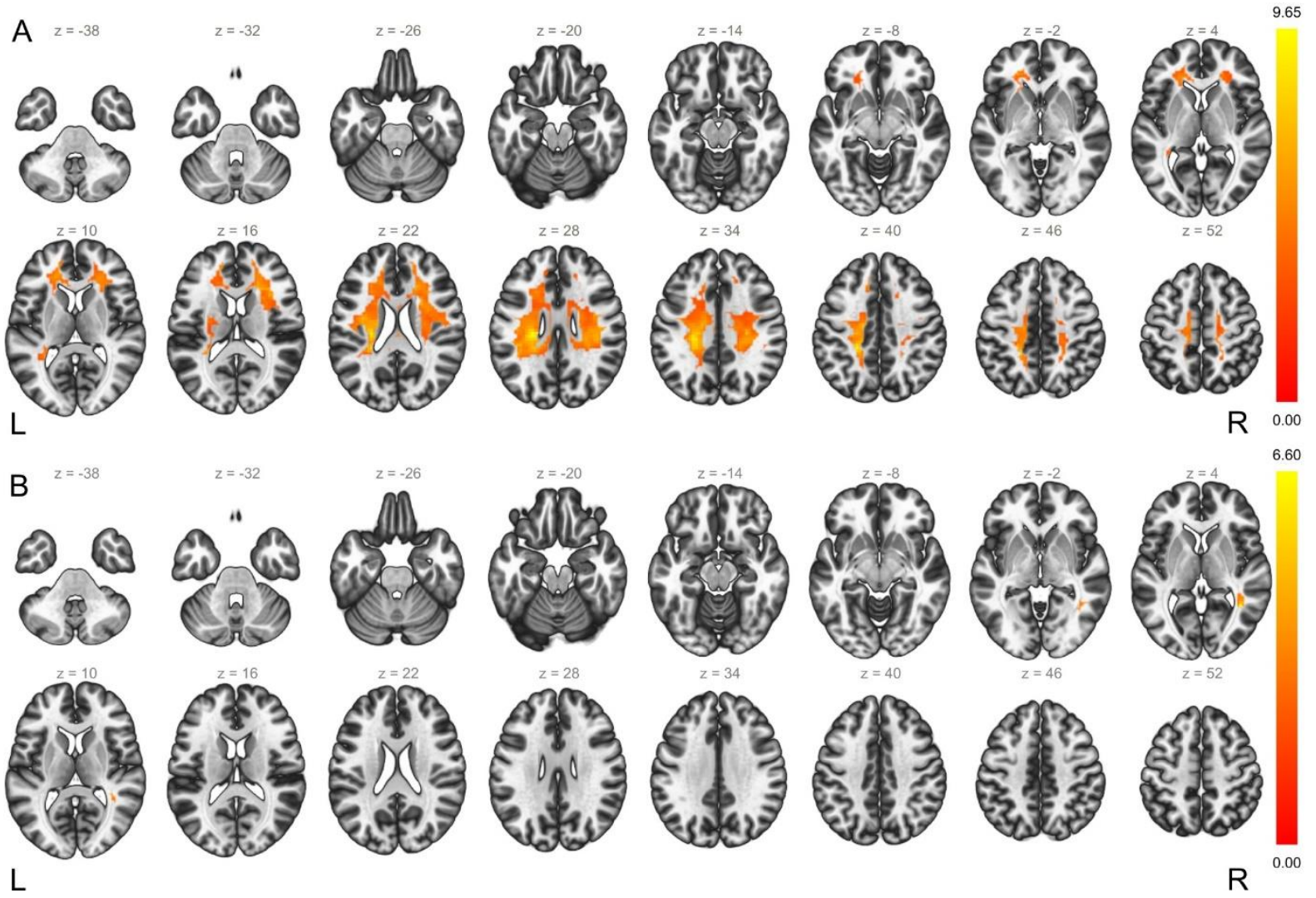

Figure 5. WM Specific ICA. (A) Independent component 8. (B) Group comparison between $\mathrm{VA}+$ and VA- patients showing a cluster predominantly composed of right superior longitudinal fasciculus.

\subsection{Regions of Interest Analysis}

\subsubsection{GM Specific Regions of Interest Analysis}

GM specific ROI analysis resulted in a single group of 2 ROI connections (Figure 6A) with significant differences in functional connectivity in the VA+ compared to VA- group $(F(2,19)$ $=16.23, \mathrm{pFDR}<0.01)$. The 2 connections were between the following resting state networks: 1) posterior division of left superior temporal gyrus (pSTG L) and anterior cerebellar network ROIs, with VA+ group showing increased functional connectivity compared to VA-group; 2) posterior division of right superior temporal gyrus (pSTG R) and posterior cerebellar network ROIs, with VA+ group showing decreased functional connectivity compared to VA- group. As ROI analysis does not provide localization, we performed a post-hoc seed-based analysis using the bilateral pSTG resting networks as seeds to possibly localise regions in the cerebellar networks. We found no significant differences between VA+ and VA-groups after correction for multiple comparisons, however results with uncorrected cluster p-values are reported here to provide some localisation. Post-hoc seed based analysis revealed two clusters (Figure 6B): 
bioRxiv preprint doi: https:/doi.org/10.1101/2021.12.03.471139; this version posted December 7, 2021. The copyright holder for this preprint (which was not certified by peer review) is the author/funder, who has granted bioRxiv a license to display the preprint in perpetuity. It is made available under aCC-BY 4.0 International license.

1) cluster 1 with MNI coordinates of cluster centre (-09, -75, -21) with regions including left cerebellum 6 and left cerebellum crus $1(\mathrm{~F}(2,19)=29.04, \mathrm{p}<0.01$ uncorrected $) ; 2)$ cluster 2 with MNI coordinates of cluster centre $(+30,-63,-21)$ and was composed of right cerebellum $6(\mathrm{~F}(2,19)=16.31, \mathrm{p}<0.05$ uncorrected $)$.
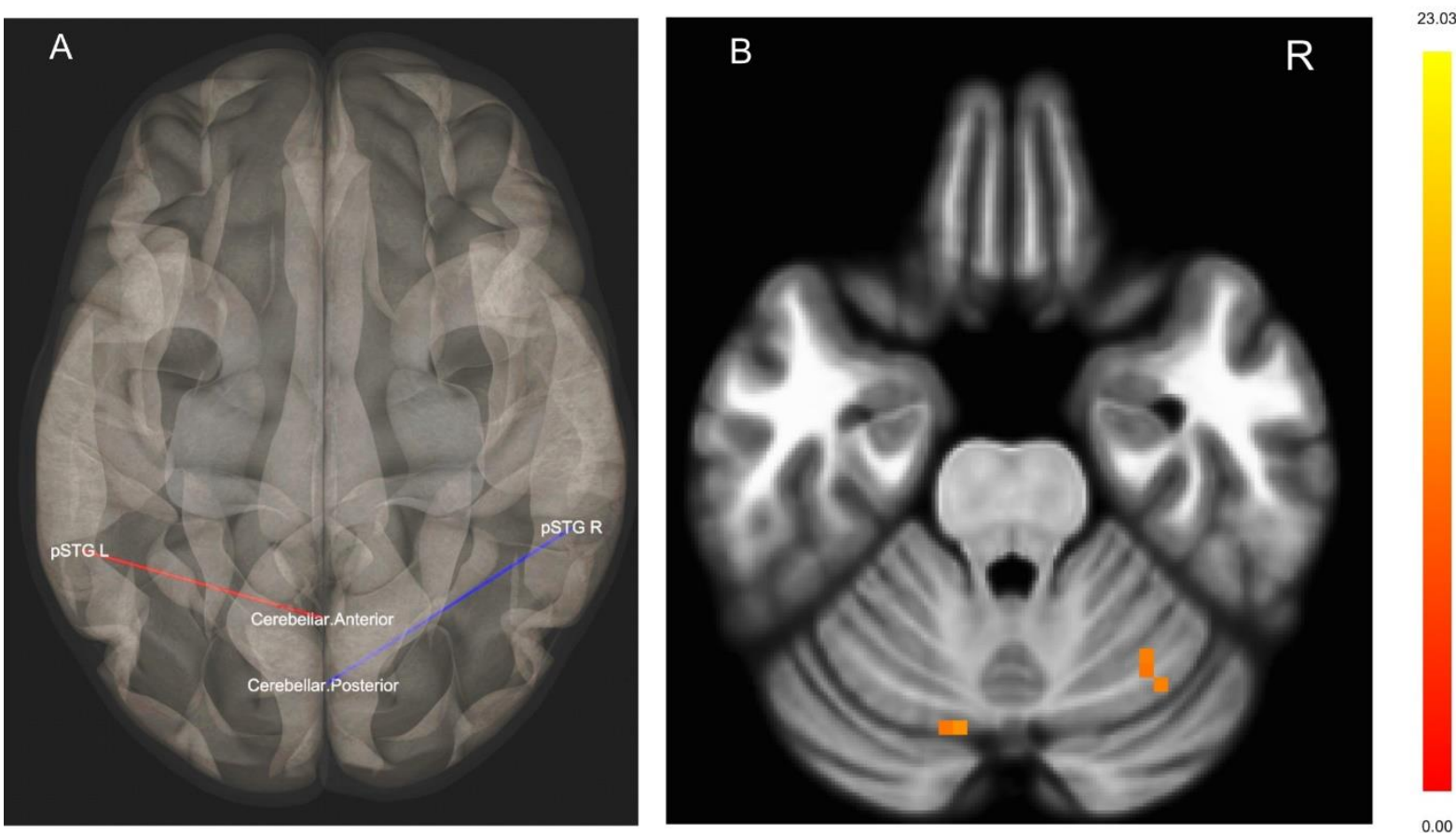

Figure 6. GM Specific ROI Analysis. (A) Bilateral connections between superior temporal gyrus and cerebellar resting state ROIs. (B) Regions within cerebellum resting state ROIs localised to left cerebellum 6 , left cerebellum crus 1 , and right cerebellum 6 using bilateral superior temporal gyri as seeds.

\subsubsection{WM Specific Regions of Interest Analysis}

WM specific ROI analysis resulted in a single group of 6 connections (Figure 7), all with increased functional connectivity in VA+ compared to VA- group (ROI mass $=66.17, \mathrm{pFDR}$ $<0.05$ ). Genu of corpus callosum (GCC), left superior longitudinal fasciculus (SLF), right SLF, left superior corona radiata (SCR), left posterior thalamic radiation (PTR), and left superior fronto-occipital fasciculus (SFOF) were all functionally connected to left anterior corona radiata (ACR), shown in Figure 7. 


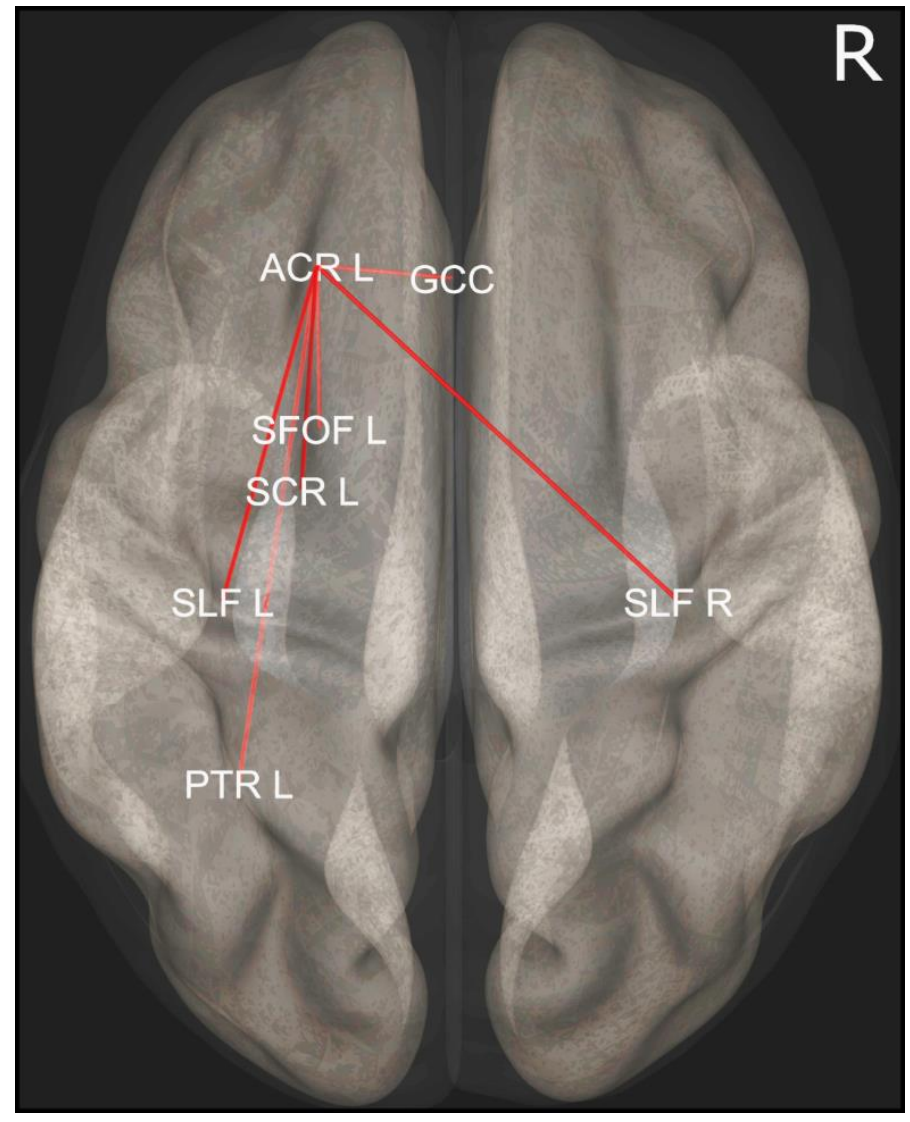

Figure 7. WM Specific ROI Analysis. (A) A group of ROI connections including genu of corpus callosum (GCC), left superior longitudinal fasciculus (SLF), right SLF, left superior corona radiata (SCR), left posterior thalamic radiation (PTR), and left superior fronto-occipital fasciculus (SFOF), all functionally linked to left Anterior Corona Radiata (ACR).

\subsection{Seed-Based Analysis}

\subsubsection{Regions with Altered Functional Connections to Vestibular Networks}

IC-13, IC-20, and IC-23, determined via GM specific ICA and composed of putative vestibular regions, were selected as seeds for seed-based analysis (supplementary Figure S1). We found that left thalamus (MNI: -12, -27, 12) had increased functional connectivity with the seed networks $(\mathrm{F}(3,18)=24.72$, pFDR $<0.05)$ in VA+ group compared to VA- group (Figure 8A). The region was localised to be a part of antero-dorsal thalamus (medial and lateral) using Melbourne Subcortex Atlas (Tian et al., 2020).

\subsubsection{Disconnection Between Temporal and Higher Order Visual Cortices}

Using bilateral lingual gyrus (LG) as seed regions, we found a cluster composed of superior and mid temporal regions with increased functional connectivity in the VA+ compared to the 
bioRxiv preprint doi: https://doi.org/10.1101/2021.12.03.471139; this version posted December 7, 2021. The copyright holder for this preprint (which was not certified by peer review) is the author/funder, who has granted bioRxiv a license to display the preprint in perpetuity. It is made available under aCC-BY 4.0 International license.

VA- group $(\mathrm{F}(2,19)=23.47, \mathrm{pFDR}<0.05)$. MNI coordinates of cluster centre were $(60,-27$, 00 ) and the cluster was composed of superior and mid temporal regions (Figure 8B).

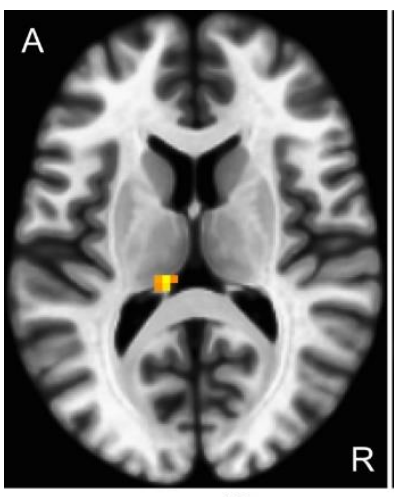

$z=12$

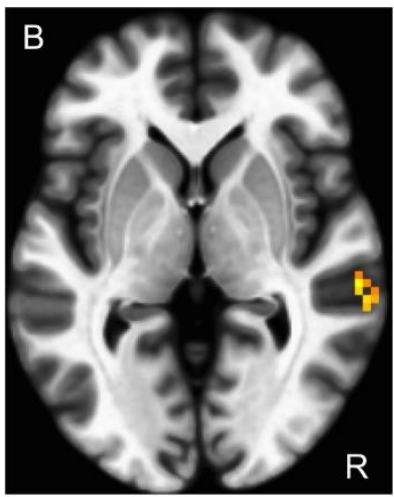

$z=0$

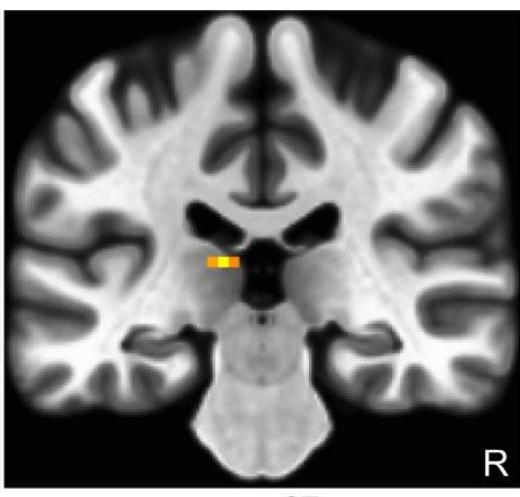

$y=-27$

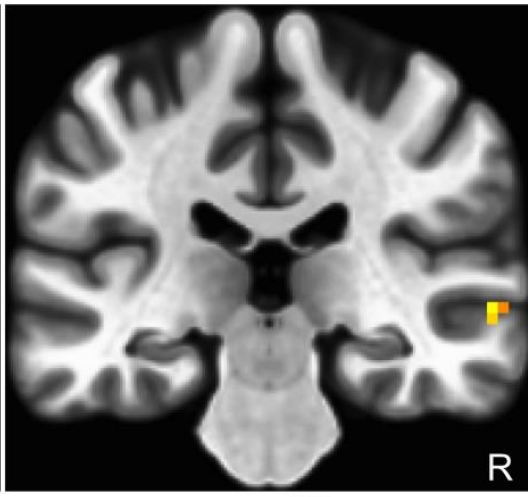

$y=-27$

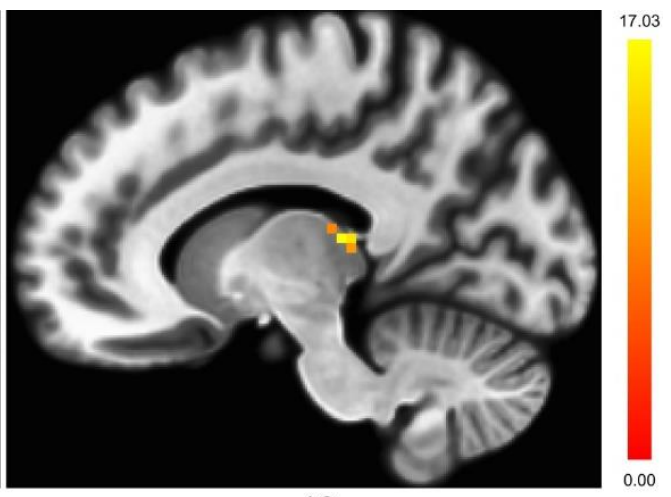

$x=12$

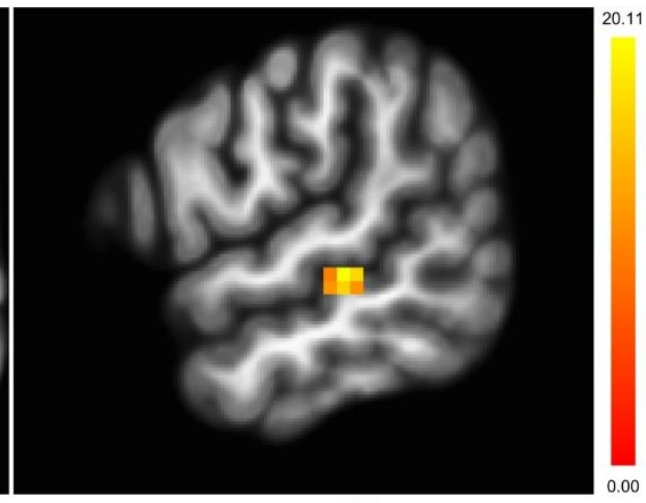

$x=60$

Figure 8. Disconnection between temporal and higher order visual cortices. (A) Left thalamus had increased functional connectivity with putative vestibular regions. (B) Increased functional connectivity of right mid- and superior-temporal regions when using bilateral lingual gyri as seed.

\subsection{Discussion}

Using DTI imaging, we previously identified that VA was linked to imbalance via damage to right ILF (Calzolari et al., 2021), however, we were unable to identify brain regions specifically explaining VA. Given our a priori hypothesis that vestibular-motion perception is mediated by multiple brain networks, we used resting-state fMRI to identify the brain regions functionally associated with vestibular agnosia. We found that vestibular agnosia is linked to: (i) increased functional connectivity in a bilateral white-matter network; (ii) increased functional connectivity in SLF; (iii) a disrupted functional link between regions mapped by ILF (Lingual gyrus (V3v/V4) and mid/superior temporal regions); (iv) a disrupted functional link between the left thalamus and resting state networks containing putative vestibular regions (PIVC, insular, and superior/mid temporal regions). 


\subsubsection{Vestibular Agnosia and White-Matter fMRI}

Using white-matter ICA, we found that vestibular agnosia was linked to a cluster predominantly composed of right superior longitudinal fasciculus (SLF). The cluster also included voxels from right posterior thalamic radiation (PTR), right retrolenticular part of internal capsule (RLIC), and right saggital stratum (SS) (Figure 5B).

A previous study has shown that electrical stimulation of the right SLF in awake neurosurgery provokes sensations of vertigo (Spena et al., 2006). Similarly, another study found that left SLF electrical stimulation evoked a more complex illusion of flying (Kahane et al., 2003). This indicates a bilateral coding of self-motion sensations via SLF. We also previously found that a large white-matter cluster in temporo-parietal region mainly composed of the SLF and more prominent in the right hemisphere, was linked to duration of vertigo sensation in healthy subjects (Nigmatullina et al., 2015). In addition to the involvement of SLF in mediating vertigo sensations, the SLF importantly, connects temporo-parietal regions with frontal cortex (Barbeau et al., 2020). This connection may mediate anterior-posterior connectivity involved in transmitting (a) bottom-up vestibular signals to frontal cortex for sensory gating; (b) recurrent activity following frontal sensory gating to posterior regions for signal amplification as hypothesized by the Global Neuronal Workspace theory (Baars, 2005; Newman et al., 1997).

The right retrolenticular internal capsule (RLIC) and right posterior thalamic radiation (PTR) have been linked with impaired balance in mild TBI and elderly adults (Gattu et al., 2016; Rosario et al., 2016), which we also found in our cohort of moderate-severe TBI (Calzolari et al., 2021). Impaired DTI parameters in RLIC are also known to be associated with impaired spatial navigation (Wu et al., 2016). Since we controlled for the variance explained by balance in our resting-state analysis, our results suggest that the RLIC may also participate in a network mediating multiple vestibular functions, such as balance, spatial navigation, and vestibular perception of self-motion, i.e. this region may function as a vestibular hub. Furthermore, we have also previously reported that right posterior thalamic radiation (PTR) and the right saggital stratum (SS) are linked to vestibular perception of self-motion in patients with impaired balance (Calzolari et al., 2021). Our results from resting-state analysis confirm the specific role of right PTR and right SS in mediating vestibular perception of self-motion. Notably the right ILF tract passes through the right SS, and right ILF intra-cortical electrical stimulation evokes yaw-plane rotational vertigo sensation (Kahane et al., 2003). 
While our ICA results show differences predominantly in the right hemisphere, the whitematter network found in ROI analysis is left lateralized with two interhemispheric connections, firstly, between the left anterior corona radiata (ACR) and right SLF and secondly a callosal connection between left ACR and genu of corpus callosum (GCC) (Figure 7). Abnormal DTI parameters in the same network of white-matter tracts shown in Figure 7 has previously been reported to be linked to impaired balance in elderly patients with small vessel disease (Rosario et al., 2016), although this study did not assess patients' ability to perceive self-motion. Notably however, there are multiple accounts of VA in patients with small vessel disease and impaired balance (Chiarovano et al., 2016; Imbaud Genieys, 2007; Seemungal, 2006), thus suggesting the involvement of overlapping albeit distinct networks, mediating multiple vestibular functions.

It is possible that the right lateralized differences from ICA could have altered the functional activation in multiple bilateral networks that are involved in self-motion perception. However, since each patient did not have purely right lateralised damage, damage to any part of these multiple bilateral networks could possibly result in altered connectivity of the networks involved in self-motion perception leading to VA. Furthermore, since the inter-network ROI findings also include corpus callosum, it is possible that damage to corpus callosum, common in TBI (Ghajari et al., 2017; Jolly et al., 2020), could have exclusively caused VA, in at least some of our subjects. Another possibility is that the vestibular processing of self-motion is predominantly right hemispheric and the left lateralized networks identified in our results are due to the overactivity in the left hemisphere, either as a compensatory response to right hemispheric function, or due to loss of right hemispheric inhibition upon left hemisphere activity, or both.

There has been only one human study assessing the effect of acute focal lesions upon the vestibular sensation of self-motion which did not find any patients with vestibular agnosia (Kaski et al., 2016). In contrast, a number of other vestibular related function are affected by focal lesions such as deficits in the visual vertical linked to focal lesions in PIVC (Baier et al., 2013) and vestibular-guided spatial disorientation with focal lesions in right temporo-parietal junction (Kaski et al., 2016). Furthermore, we have previously shown that the duration of vestibular-motion perception is linked to a widespread bilateral white-matter network (Nigmatullina et al., 2015). Indeed tractography studies indicate a bihemispheric vestibular network linked via the corpus callosum (Kirsch et al., 2016; Wirth et al., 2018). Accordingly, our finding suggesting that damaged bilateral white matter network mediates vestibular agnosia 
seem more likely than that of a compensatory network, although the two possibilities are not mutually exclusive.

\subsubsection{Vestibular Agnosia and Grey-Matter fMRI}

Using ICA we found that a decreased activity, of the right Intra Calcarine Cortex (ICC) in a bilateral mid-temporal resting-state network, and an overactivation of left frontal pole in a bilateral rostral prefrontal cortical network, were linked to vestibular agnosia.

Various sites in the mid-temporal gyrus have previously been shown to produce rotational sensation of self-motion induced by intra-cortical electrical stimulation (Kahane et al., 2003) and are also activated in response to non-invasive galvanic stimulation (Della-Justina et al., 2014). Additionally, the calcarine region is often shown to be linked with visual motion and visuo-vestibular interaction (Cha \& Chakrapani, 2015; Della-Justina et al., 2014). Since the ILF connects the calcarine and temporal regions (Latini et al., 2017; Panesar et al., 2018), the deactivation of the ICC within a bilateral mid-temporal resting-state network might be due to the damage to the ILF, which we previously concluded in this cohort via DTI (Calzolari et al., 2021).

Rostral prefrontal cortex (RPFC) is well established as a region associated with the integration of information and creating an internal representation, which then allows attending to stimuli (Stocco et al., 2012). There are different hypotheses explaining the possible role of the RPFC. The 'Gateway hypothesis' suggests the existence of an internal representation independent of external stimuli (equivalent to a Bayesian 'prior') and an internal representation linked to environmental stimuli (equivalent to a Bayesian 'posterior') and RPFC mediates the interaction between both internal representations (Burgess et al., 2007). Moreover, the left RPFC rather than right is linked to higher order processing when integrating multiple representations (Bunge et al., 2009). In contrast to the integration hypotheses, an 'ignition' hypothesis of perception mediated by the prefrontal cortex, suggests that a stimulus must surpass a threshold to trigger recurrent circuits that amplify and broadcast the initial signal to associated brain regions and in so doing mediate conscious perception of the signal (Del Cul et al., 2007, 2009). Our finding that VA+ patients show increased activation in the left frontal pole within the RPFC does not however, allow us to discriminate between the integration or ignition hypotheses.

While looking at the inter-network differences using ROI approach, we found bilateral functional connections between the superior temporal gyri and cerebellar resting-state 
networks. There was a relative increase in activation of left hemispheric connections and a decrease in right hemisphere connections, for VA+ patients when compared to VA- patients.

Since resting-state networks do not provide any localization of regions per se, we localized cerebellar regions inside the cerebellar resting-state network using a seed-based analysis. There were no statistically significant differences, however, uncorrected findings (for multiple comparisons and hence require a conservative interpretation) showed bilateral functional connections from temporal gyri localized to the left cerebellum VI/Crus I and right cerebellum VI. The left cerebellum VI has been shown to activate in response to vestibular, visual, and simultaneous stimulations of both modalities, however, activation in response to vestibular stimulation is found to be significantly higher than the simultaneous stimulation of both modalities pointing to the vestibular specific role of left cerebellum VI (Della-Justina et al., 2014). While right cerebellum VI has not been reported previously to have a vestibular role, our results suggest the presence of functional link of the bilateral temporal gyri and the cerebellar resting-state networks, pointing to the existence of bilateral connections from temporal gyri to the cerebellum.

\subsubsection{Altered Connectivity of Vestibular Networks Linked to Vestibular Agnosia}

Based upon the current understanding of the human vestibular cortical system, bilateral temporo-parietal and insular cortex are usually considered important for vestibular processing with parieto-insular vestibular cortex (PIVC), and posterior insular cortex (PIC) considered the core vestibular regions (Wirth et al., 2018). To improve generalizability, we adopted a networkbased approach and used the resting state networks composed of these vestibular regions (PIVC and PIC) in a seed-based analysis. We found that patients with vestibular agnosia (VA+) had an overactivated functional link between the left thalamus and the vestibular resting-state networks.

Notably, the MNI coordinates of the left thalamus localized to the medial and lateral parts of the antero-dorsal thalamus, of potential relevance since the thalamus is an important vestibular structure and is considered a 'gateway' for vestibular information (Dieterich et al., 2005) with bidirectional projections linking brainstem and cortical vestibular regions (Kirsch et al., 2016). Previous tractography studies have shown that PIVC and PIC have structural connections with the thalamus in each hemisphere (Kirsch et al., 2016; Wirth et al., 2018). Most of the previous studies have reported posterolateral parts of thalamus to be important centers for vestibular processing including ventral posterior lateral nucleus (VPL), ventral posterior medial (VPM) 
bioRxiv preprint doi: https://doi.org/10.1101/2021.12.03.471139; this version posted December 7, 2021. The copyright holder for this preprint (which was not certified by peer review) is the author/funder, who has granted bioRxiv a license to display the preprint in perpetuity. It is made available under aCC-BY 4.0 International license.

nucleus, lateral posterior (LP) nucleus, and ventral lateral posterior or ventro-intermediate nucleus (VLP/Vim) (Baier et al., 2016, 2017; Dieterich et al., 2005; Dieterich \& Brandt, 1993). However, it is important to note that most of these findings have been reported for visual tilt perception tasks and do not provide much information about self-motion perception processing.

Reports on thalamic involvement in vestibular velocity processing are relatively sparse and are not limited to the posterolateral thalamus. Old reports in human electrical stimulation studies show the involvement of the ventro-intermediate (Vim) nucleus in processing of rotational sensation of body and head (Hawrylyshyn et al., 1978; Kirsch et al., 2016; Tasker et al., 1982). The processing of head angular velocity is shown to be linked with head direction cells in the anterodorsal thalamus (Shinder \& Taube, 2011) and brain-stem (Sharp et al., 2001), implying a possible involvement of head direction cells within anterior thalamic nuclei in velocity processing, which we observed in our findings.

Anterior thalamic nuclei are thought to have projections to hippocampus (Brandt et al., 2005) possibly via the posterior parietal cortex (Smith, 1997), which, however, has not been explicitly confirmed in humans (Lopez \& Blanke, 2011). While vestibulo-hippocampal interactions are typically linked with spatial processing, a problem in velocity integration may also result in abnormal spatial processing and thus belying the potential relevance of velocity processing in anterior thalamic nuclei to vestibular spatial processing.

\subsubsection{Disconnection Between Higher Order Visual Cortices and Temporal Regions}

We found that VA+ subjects had a disruption of functional link between right lingual gyrus and right mid-temporal gyrus indicating disrupted functional projections from higher order visual cortex in these patients. This disruption was perhaps predictable given the known connectivity of the ILF linking the temporal and lingual gyri (Latini et al., 2017; Panesar et al., 2018) and our previous report of ILF disruption in VA+ patients (Calzolari et al., 2021).

The lingual gyrus appears to be an important vestibular processing hub that is also involved in visuo-vestibular motion processing (Della-Justina et al., 2014; Roberts et al., 2017). In particular, the lingual gyrus is activated by visual, vestibular, and visuo-vestibular motion stimulation, however, its activation for isolated vestibular stimulation is greater than that for simultaneous visuo-vestibular stimulation indicating its prominent role in vestibular specific signal processing (Della-Justina et al., 2014; Roberts et al., 2017). Indeed intra-cortical electrical stimulation of the lingual gyrus evoked a yaw-plane sensation of self-motion (Kahane et al., 2003). Hence, in addition to previous data implicating the lingual gyrus in visuo- 
vestibular motion processing, our data further suggest that a right hemisphere network involving the lingual gyrus and mid-temporal regions connected via the right ILF, is involved in self-motion processing.

\subsection{Limitations}

Vestibular agnosia can currently only be objectively identified via self-motion perception testing in a rotatory-chair in dark. Moreover, having vestibular agnosia is a continuum with mild or borderline cases may or may not be classified as having vestibular agnosia. Since this is one of the first studies looking at link between vestibular agnosia and resting-state functional networks, our focus was on optimizing the sensitivity of the analysis while keeping an adequate specificity. Another limitation of the study is the small sample size. This was partly due to a limited number of vestibular agnosia patients as well as due to some unforeseeable scanning parameter mismatch issues. Vestibular agnosia is known to impact elderly people and neurodegeneration patients, however an advantage of our patient group however was their relative young age and good premorbid health excluding incipient neurodegenerative disease (given our stringent exclusion criteria), hence the findings reported herein were overwhelmingly related to the acute TBI and not to other chronic underlying disease.

\subsection{Conclusion}

Our data provide the first evidence linking resting-state functional networks to vestibular agnosia. More specifically, we show altered connectivity in a bihemispheric white matter network, involvement of putative vestibular regions i.e. PIVC, insular, superior \& midtemporal regions, and disconnection between higher order visual cortices involved in motion processing and the superior- and mid-temporal regions in right hemisphere. The involvement of left prefrontal regions also points to the possibility of an "ignition" failure in the loss of vertigo perception. Our findings thus suggest that vestibular agnosia is not linked to a focal brain abnormality but is linked to a multi-network, inter- and intra-network, dysfunction.

\section{Declaration of Competing Interests}

The authors declare that they have no competing interests.

\section{Acknowledgements}

We would like to thank our patient and healthy volunteers for their participation and for helping us improve the traumatic brain injury patients' care. We are also grateful to the major trauma ward teams at St Mary's Hospital and King's College Hospital London for their help with 
recruitment and assessment. We are also very grateful to The Imperial Health Charity who provided important kickstarter funding that enabled us to obtain research council funding for this project.

\section{Funding}

The Medical Research Council (MRC). The Imperial Health Charity, The NIHR Imperial Biomedical Research Centre, NIHR Clinical Doctoral Research Fellowship, The US Department of Defense - Congressionally Directed Medical Research Program (CDMRP). The Jon Moulton Charity Trust.

\section{Data and code availability statements}

Raw data that support the findings of this study are available from the corresponding author, upon reasonable request. The request would require a formal data sharing agreement, approval from the requesting researcher's local ethics committee, a formal project outline, and discussion about authorship on any research output from the shared data.

\section{Credit author statement}

Zaeem Hadi: Investigation, Methodology, Software, Formal analysis, Visualization, Writing - Original Draft. Yuscah Pondeca: Investigation, Methodology, Writing - review and editing. Elena Calzolari: Investigation, Methodology, Writing - review and editing. Mariya Chepisheva: Investigation, Writing - review and editing. Rebecca M Smith: Investigation, Methodology, Writing - review and editing. Mohammad Mahmud: Investigation, Methodology, Writing - review and editing. Heiko Rust: Investigation, Methodology, Writing - review and editing. David J Sharp: Supervision, Writing - review and editing. Barry M Seemungal: Conceptualization, Investigation, Project administration, Funding acquisition, Resources, Supervision, Writing - review and editing. 


\section{References}

Baars, B. J. (2005). Global workspace theory of consciousness: toward a cognitive neuroscience of human experience. Progress in Brain Research, 150, 45-53. https://doi.org/10.1016/S0079-6123(05)50004-9

Baier, B., Conrad, J., Stephan, T., Kirsch, V., Vogt, T., Wilting, J., Müller-Forell, W., \& Dieterich, M. (2016). Vestibular thalamus Two distinct graviceptive pathways. Neurology, 86(2), 134-140. https://doi.org/10.1212/WNL.0000000000002238

Baier, B., Conrad, J., Zu Eulenburg, P., Best, C., Müller-Forell, W., Birklein, F., \& Dieterich, M. (2013). Insular strokes cause no vestibular deficits. Stroke, 44(9), 2604-2606. https://doi.org/10.1161/STROKEAHA.113.001816

Baier, B., Vogt, T., Rohde, F., Cuvenhaus, H., Conrad, J., \& Dieterich, M. (2017). Deep brain stimulation of the nucleus ventralis intermedius: a thalamic site of graviceptive modulation. Brain Structure and Function, 222(1), 645-650. https://doi.org/10.1007/s00429-015-1157-x

Barbeau, E. B., Descoteaux, M., \& Petrides, M. (2020). Dissociating the white matter tracts connecting the temporo-parietal cortical region with frontal cortex using diffusion tractography. Scientific Reports 2020 10:1, 10(1), 1-13. https://doi.org/10.1038/s41598020-64124-y

Behzadi, Y., Restom, K., Liau, J., \& Liu, T. T. (2007). A component based noise correction method (CompCor) for BOLD and perfusion based fMRI. NeuroImage, 37(1), 90-101. https://doi.org/10.1016/j.neuroimage.2007.04.042

Branco, P., Seixas, D., \& Castro, S. L. (2020). Mapping language with resting-state functional magnetic resonance imaging: A study on the functional profile of the language network. Human Brain Mapping, 41(2), 545-560. https://doi.org/10.1002/HBM.24821

Branco, P., Seixas, D., Deprez, S., Kovacs, S., Peeters, R., Castro, S. L., \& Sunaert, S. (2016). Resting-State Functional Magnetic Resonance Imaging for Language Preoperative Planning. Frontiers in Human Neuroscience, O(FEB2016), 11. https://doi.org/10.3389/FNHUM.2016.00011

Brandt, T., Schautzer, F., Hamilton, D. A., Brüning, R., Markowitsch, H. J., Kalla, R., Darlington, C., Smith, P., \& Strupp, M. (2005). Vestibular loss causes hippocampal 
atrophy and impaired spatial memory in humans. Brain, 128(11), 2732-2741. https://doi.org/10.1093/brain/awh617

Bullmore, E. T., Suckling, J., Overmeyer, S., Rabe-Hesketh, S., Taylor, E., \& Brammer, M. J. (1999). Global, voxel, and cluster tests, by theory and permutation, for a difference between two groups of structural mr images of the brain. IEEE Transactions on Medical Imaging, 18(1), 32-42. https://doi.org/10.1109/42.750253

Bunge, S. A., Helskog, E. H., \& Wendelken, C. (2009). Left, but not right, rostrolateral prefrontal cortex meets a stringent test of the relational integration hypothesis. In NeuroImage (Vol. 46, Issue 1, pp. 338-342). Academic Press Inc. https://doi.org/10.1016/j.neuroimage.2009.01.064

Burgess, P. W., Dumontheil, I., \& Gilbert, S. J. (2007). The gateway hypothesis of rostral prefrontal cortex (area 10) function. Trends in Cognitive Sciences, 11(7), 290-298. https://doi.org/10.1016/j.tics.2007.05.004

Calzolari, E., Chepisheva, M., Smith, R. M., Mahmud, M., Hellyer, P. J., Tahtis, V., Arshad, Q., Jolly, A., Wilson, M., Rust, H., Sharp, D. J., \& Seemungal, B. M. (2021). Vestibular agnosia in traumatic brain injury and its link to imbalance. Brain : A Journal of Neurology, 144(1), 128-143. https://doi.org/10.1093/brain/awaa386

Cha, Y.-H., \& Chakrapani, S. (2015). Voxel Based Morphometry Alterations in Mal de Debarquement Syndrome. PLOS ONE, 10(8), e0135021. https://doi.org/10.1371/journal.pone.0135021

Chiarovano, E., Vidal, P. P., Magnani, C., Lamas, G., Curthoys, I. S., \& de Waele, C. (2016). Absence of rotation perception during warm water caloric irrigation in some seniors with postural instability. Frontiers in Neurology, 7(JAN). https://doi.org/10.3389/fneur.2016.00004

Del Cul, A., Baillet, S., \& Dehaene, S. (2007). Brain dynamics underlying the nonlinear threshold for access to consciousness. PLoS Biology, 5(10), 2408-2423. https://doi.org/10.1371/journal.pbio.0050260

Del Cul, A., Dehaene, S., Reyes, P., Bravo, E., \& Slachevsky, A. (2009). Causal role of prefrontal cortex in the threshold for access to consciousness. Brain, 132(9), 2531-2540. https://doi.org/10.1093/brain/awp111 
Della-Justina, H. M., Gamba, H. R., Lukasova, K., Nucci-da-Silva, M. P., Winkler, A. M., \& Amaro, E. (2014). Interaction of brain areas of visual and vestibular simultaneous activity with fMRI. Experimental Brain Research, 233(1), 237-252. https://doi.org/10.1007/s00221-014-4107-6

Dieterich, M., Bartenstein, P., Spiegel, S., Bense, S., Schwaiger, M., \& Brandt, T. (2005). Thalamic infarctions cause side-specific suppression of vestibular cortex activations. Brain, 128(9), 2052-2067. https://doi.org/10.1093/brain/awh551

Dieterich, M., \& Brandt, T. (1993). Thalamic infarctions: Differential effects on vestibular function in the roll plane (35 patients). Neurology, 43(9), 1732-1740. https://doi.org/10.1212/wnl.43.9.1732

Ding, Z., Newton, A. T., Xu, R., Anderson, A. W., Morgan, V. L., \& Gore, J. C. (2013). Spatiotemporal correlation tensors reveal functional structure in human brain. PLoS ONE, 8(12), 82107. https://doi.org/10.1371/journal.pone.0082107

Ding, Z., Xu, R., Bailey, S. K., Wu, T. L., Morgan, V. L., Cutting, L. E., Anderson, A. W., \& Gore, J. C. (2016). Visualizing functional pathways in the human brain using correlation tensors and magnetic resonance imaging. Magnetic Resonance Imaging, 34(1), 8-17. https://doi.org/10.1016/j.mri.2015.10.003

Eklund, A., Knutsson, H., \& Nichols, T. E. (2019). Cluster failure revisited: Impact of first level design and physiological noise on cluster false positive rates. Human Brain Mapping, 40(7), 2017-2032. https://doi.org/10.1002/hbm.24350

Fraser, L. M., Stevens, M. T., Beyea, S. D., \& D’Arcy, R. C. N. (2012). White versus gray matter: fMRI hemodynamic responses show similar characteristics, but differ in peak amplitude. BMC Neuroscience, 13(1), 91. https://doi.org/10.1186/1471-2202-13-91

Gattu, R., Akin, F. W., Cacace, A. T., Hall, C. D., Murnane, O. D., \& Haacke, E. M. (2016). Vestibular, balance, microvascular and white matter neuroimaging characteristics of blast injuries and mild traumatic brain injury: Four case reports. Brain Injury, 30(12), 15011514. https://doi.org/10.1080/02699052.2016.1219056

Ghajari, M., Hellyer, P. J., \& Sharp, D. J. (2017). Computational modelling of traumatic brain injury predicts the location of chronic traumatic encephalopathy pathology. Brain, 140(2), 333-343. https://doi.org/10.1093/brain/aww317 
Hawrylyshyn, P. A., Rubin, A. M., Tasker, R. R., Organ, L. W., \& Fredrickson, J. M. (1978). Vestibulothalamic projections in man. A sixth primary sensory pathway. Journal of Neurophysiology, 41(2), 394-401. https://doi.org/10.1152/jn.1978.41.2.394

Huang, Y., Bailey, S. K., Wang, P., Cutting, L. E., Gore, J. C., \& Ding, Z. (2018). Voxel-wise detection of functional networks in white matter. NeuroImage, 183, 544-552. https://doi.org/10.1016/j.neuroimage.2018.08.049

Huang, Y., Yang, Y., Hao, L., Hu, X., Wang, P., Ding, Z., Gao, J. H., \& Gore, J. C. (2020). Detection of functional networks within white matter using independent component analysis. NeuroImage, 222, 117278. https://doi.org/10.1016/j.neuroimage.2020.117278

Imbaud Genieys, S. (2007). Vertigo, dizziness and falls in the elderly. Annales d'OtoLaryngologie et de Chirurgie Cervico-Faciale, 124(4), 189-196. https://doi.org/10.1016/j.aorl.2007.04.003

Jafri, M. J., Pearlson, G. D., Stevens, M., \& Calhoun, V. D. (2008). A method for functional network connectivity among spatially independent resting-state components in schizophrenia. NeuroImage, 39(4), 1666-1681. https://doi.org/10.1016/j.neuroimage.2007.11.001

Jolly, A. E., Bălăeţ, M., Azor, A., Friedland, D., Sandrone, S., Graham, N. S. N., Zimmerman, K., \& Sharp, D. J. (2020). Detecting axonal injury in individual patients after traumatic brain injury. Brain. https://doi.org/10.1093/brain/awaa372

Kahane, P., Hoffmann, D., Minotti, L., \& Berthoz, A. (2003). Reappraisal of the Human Vestibular Cortex by Cortical Electrical Stimulation Study. Annals of Neurology, 54(5), 615-624. https://doi.org/10.1002/ana.10726

Kaski, D., Quadir, S., Nigmatullina, Y., Malhotra, P. A., Bronstein, A. M., \& Seemungal, B. M. (2016). Temporoparietal encoding of space and time during vestibular-guided orientation. Brain, 139(2), 392-403. https://doi.org/10.1093/brain/awv370

Kirsch, V., Keeser, D., Hergenroeder, T., Erat, O., Ertl-Wagner, B., Brandt, T., \& Dieterich, M. (2016). Structural and functional connectivity mapping of the vestibular circuitry from human brainstem to cortex. Brain Structure and Function, 221(3), 1291-1308. https://doi.org/10.1007/s00429-014-0971-x

Latini, F., Mårtensson, J., Larsson, E. M., Fredrikson, M., Åhs, F., Hjortberg, M., Aldskogius, 
H., \& Ryttlefors, M. (2017). Segmentation of the inferior longitudinal fasciculus in the human brain: A white matter dissection and diffusion tensor tractography study. Brain Research, 1675, 102-115. https://doi.org/10.1016/j.brainres.2017.09.005

Li, M., Gao, Y., Gao, F., Anderson, A. W., Ding, Z., \& Gore, J. C. (2020). Functional engagement of white matter in resting-state brain networks. NeuroImage, 220, 117096. https://doi.org/10.1016/j.neuroimage.2020.117096

Lopez, C., \& Blanke, O. (2011). The thalamocortical vestibular system in animals and humans. In Brain Research Reviews (Vol. 67, Issues 1-2, pp. 119-146). Elsevier. https://doi.org/10.1016/j.brainresrev.2010.12.002

Marussich, L., Lu, K. H., Wen, H., \& Liu, Z. (2017). Mapping white-matter functional organization at rest and during naturalistic visual perception. NeuroImage, 146, 11281141. https://doi.org/10.1016/j.neuroimage.2016.10.005

Mori, S., Oishi, K., Jiang, H., Jiang, L., Li, X., Akhter, K., Hua, K., Faria, A. V., Mahmood, A., Woods, R., Toga, A. W., Pike, G. B., Neto, P. R., Evans, A., Zhang, J., Huang, H., Miller, M. I., van Zijl, P., \& Mazziotta, J. (2008). Stereotaxic white matter atlas based on diffusion tensor imaging in an ICBM template. NeuroImage, 40(2), 570-582. https://doi.org/10.1016/j.neuroimage.2007.12.035

Murphy, K., Birn, R. M., Handwerker, D. A., Jones, T. B., \& Bandettini, P. A. (2009). The impact of global signal regression on resting state correlations: Are anti-correlated networks introduced? $\quad$ NeuroImage, 44(3), 893-905. https://doi.org/10.1016/j.neuroimage.2008.09.036

Newman, J., Baars, B. J., \& Cho, S. B. (1997). A Neural Global Workspace Model for Conscious Attention. Neural Networks, 10(7), 1195-1206. https://doi.org/10.1016/S0893-6080(97)00060-9

Nichols, T. E. (2012). Multiple testing corrections, nonparametric methods, and random field theory. https://doi.org/10.1016/J.NEUROIMAGE.2012.04.014

Nigmatullina, Y., Hellyer, P. J., Nachev, P., Sharp, D. J., \& Seemungal, B. M. (2015). The neuroanatomical correlates of training-related perceptuo-reflex uncoupling in dancers. Cerebral Cortex, 25(2), 554-562. https://doi.org/10.1093/cercor/bht266 
Ogawa, S., Tank, D. W., Menon, R., Ellermann, J. M., Kim, S. G., Merkle, H., \& Ugurbil, K. (1992). Intrinsic signal changes accompanying sensory stimulation: Functional brain mapping with magnetic resonance imaging. Proceedings of the National Academy of Sciences of the United States of America, 89(13), 5951-5955. https://doi.org/10.1073/pnas.89.13.5951

Oishi, K., Zilles, K., Amunts, K., Faria, A., Jiang, H., Li, X., Akhter, K., Hua, K., Woods, R., Toga, A. W., Pike, G. B., Rosa-Neto, P., Evans, A., Zhang, J., Huang, H., Miller, M. I., van Zijl, P. C. M., Mazziotta, J., \& Mori, S. (2008). Human brain white matter atlas: Identification and assignment of common anatomical structures in superficial white matter. NeuroImage, 43(3), 447-457. https://doi.org/10.1016/j.neuroimage.2008.07.009

Panesar, S. S., Yeh, F. C., Jacquesson, T., Hula, W., \& Fernandez-Miranda, J. C. (2018). A quantitative tractography study into the connectivity segmentation laterality of the human inferior longitudinal fasciculus. Frontiers in Neuroanatomy, 12. https://doi.org/10.3389/fnana.2018.00047

Peer, M., Nitzan, M., Bick, A. S., Levin, N., \& Arzy, S. (2017). Evidence for functional networks within the human brain's white matter. Journal of Neuroscience, 37(27), 63946407. https://doi.org/10.1523/JNEUROSCI.3872-16.2017

Power, J. D., Barnes, K. A., Snyder, A. Z., Schlaggar, B. L., \& Petersen, S. E. (2012). Spurious but systematic correlations in functional connectivity MRI networks arise from subject $\begin{array}{lll}\text { motion. } & \text { NeuroImage, } & \text { 2142-2154. }\end{array}$ https://doi.org/10.1016/j.neuroimage.2011.10.018

Roberts, R. E., Ahmad, H., Arshad, Q., Patel, M., Dima, D., Leech, R., Seemungal, B. M., Sharp, D. J., \& Bronstein, A. M. (2017). Functional neuroimaging of visuo-vestibular interaction. Brain Structure and Function, 222(5), 2329-2343. https://doi.org/10.1007/s00429-016-1344-4

Rosario, B. L., Rosso, A. L., Aizenstein, H. J., Harris, T., Newman, A. B., Satterfield, S., Studenski, S. A., Yaffe, K., \& Rosano, C. (2016). Cerebral white matter and slow gait: Contribution of hyperintensities and normal-appearing parenchyma. Journals of Gerontology - Series A Biological Sciences and Medical Sciences, 71(7), 968-973. https://doi.org/10.1093/gerona/glv224

Seemungal, B. M. (2006). The mechanisms and loci of human vestibular perception 
[University of London]. https://discovery.ucl.ac.uk/id/eprint/1445054/

Seemungal, B. M. (2016). Screening for BPPV in falls: An easy but big clinical "win." BMJ (Online), 353. https://doi.org/10.1136/bmj.i3004

Seemungal, B. M., Gunaratne, I. A., Fleming, I. O., Gresty, M. A., \& Bronstein, A. M. (2004). Perceptual and nystagmic thresholds of vestibular function in yaw. Journal of Vestibular Research, 14(6), 461-466.

Seemungal, B. M., Rizzo, V., Gresty, M. A., Rothwell, J. C., \& Bronstein, A. M. (2008). Posterior parietal rTMS disrupts human Path Integration during a vestibular navigation task. Neuroscience Letters, 437(2), 88-92. https://doi.org/10.1016/j.neulet.2008.03.067

Sharp, P. E., Tinkelman, A., \& Cho, J. (2001). Angular velocity and head direction signals recorded from the dorsal tegmental nucleus of gudden in the rat: Implications for path integration in the head direction cell circuit. Behavioral Neuroscience, 115(3), 571-588. https://doi.org/10.1037/0735-7044.115.3.571

Shinder, M. E., \& Taube, J. S. (2011). Active and passive movement are encoded equally by head direction cells in the anterodorsal thalamus. Journal of Neurophysiology, 106(2), 788-800. https://doi.org/10.1152/jn.01098.2010

Smith, P. F. (1997). Vestibular-hippocampal interactions. Hippocampus, 7(5), 465-471. https://doi.org/10.1002/(SICI)1098-1063(1997)7:5<465::AID-HIPO3>3.0.CO;2-G

Spena, G., Gatignol, P., Capelle, L., \& Duffau, H. (2006). Superior longitudinal fasciculus subserves vestibular network in humans. NeuroReport, 17(13), 1403-1406. https://doi.org/10.1097/01.wnr.0000223385.49919.61

Stocco, A., Lebiere, C., O’Reilly, R. C., \& Anderson, J. R. (2012). Distinct contributions of the caudate nucleus, rostral prefrontal cortex, and parietal cortex to the execution of instructed tasks. Cognitive, Affective and Behavioral Neuroscience, 12(4), 611-628. https://doi.org/10.3758/s13415-012-0117-7

Tasker, R. R., Organ, L. W., \& Hawrylyshyn, P. (1982). Investigation of the surgical target for alleviation of involuntary movement disorders. Applied Neurophysiology, 45(3), 261274. https://doi.org/10.1159/000101610

Tian, Y., Margulies, D. S., Breakspear, M., \& Zalesky, A. (2020). Topographic organization of the human subcortex unveiled with functional connectivity gradients. Nature 
Neuroscience, 23(11), 1421-1432. https://doi.org/10.1038/s41593-020-00711-6

Tie, Y., Rigolo, L., Norton, I. H., Huang, R. Y., Wu, W., Orringer, D., Mukundan, S., \& Golby, A. J. (2014). Defining language networks from resting-state fMRI for surgical planninga feasibility study. Human Brain Mapping, 35(3), 1018-1030. https://doi.org/10.1002/HBM.22231

Whitfield-Gabrieli, S., \& Nieto-Castanon, A. (2012). Conn: A Functional Connectivity Toolbox for Correlated and Anticorrelated Brain Networks. Brain Connectivity, 2(3), 125-141. https://doi.org/10.1089/brain.2012.0073

Wirth, A. M., Frank, S. M., Greenlee, M. W., \& Beer, A. L. (2018). White Matter Connectivity of the Visual-Vestibular Cortex Examined by Diffusion-Weighted Imaging. Brain Connectivity, 8(4), 235-244. https://doi.org/10.1089/brain.2017.0544

Wu, Y. F., Wu, W. B., Liu, Q. P., He, W. W., Ding, H., Nedelska, Z., Hort, J., Zhang, B., \& $\mathrm{Xu}, \mathrm{Y}$. (2016). Presence of lacunar infarctions is associated with the spatial navigation impairment in patients with mild cognitive impairment: A DTI study. Oncotarget, 7(48), 78310-78319. https://doi.org/10.18632/oncotarget.13409

Yousif, N., Bhatt, H., Bain, P. G., Nandi, D., \& Seemungal, B. M. (2016). The effect of pedunculopontine nucleus deep brain stimulation on postural sway and vestibular perception. European Journal of Neurology, 23(3), 668-670. https://doi.org/10.1111/ene.12947

Yushkevich, P. A., Piven, J., Hazlett, H. C., Smith, R. G., Ho, S., Gee, J. C., \& Gerig, G. (2006). User-guided 3D active contour segmentation of anatomical structures: Significantly improved efficiency and reliability. NeuroImage, 31(3), 1116-1128. https://doi.org/10.1016/j.neuroimage.2006.01.015

Zhang, H., Nichols, T. E., \& Johnson, T. D. (2009). Cluster mass inference via random field theory.

NeuroImage, 44(1), $51-61$. https://doi.org/10.1016/J.NEUROIMAGE.2008.08.017 